\title{
Dynamic modeling of the morphology of multiphase waterborne polymer particles
}

\author{
Elena Akhmatskaya • José M. Asua
}

Received: 13 March 2012 / Revised: 27 June 2012 / Accepted: 15 July 2012 / Published online: 29 July 2012

(C) Springer-Verlag 2012

\begin{abstract}
Multiphase waterborne polymer particles provide advantages in more demanding applications, and their performance depends on particle morphology. Currently, no dynamic model for the prediction of the development of the morphology of multiphase latex particles is available. In this work, a model was developed for the prediction of the dynamic development of the morphology of multiphase waterborne systems, such as polymer-polymer and polymerpolymer-inorganic hybrids.
\end{abstract}

Keywords Waterborne multiphase particles · Dynamic modeling · Development of particle morphology ·

Polymer-polymer hybrid · Polymer-polymer-inorganic hybrid

\section{Introduction}

Waterborne polymers are used in a wide range of applications including synthetic rubber, paints, adhesives, additives in paper and textiles, leather treatment, impact modifiers for plastic matrices, additives for constructing materials, cosmetics, flocculants, diagnostic tests, and drug delivery [1-3]. Although homogeneous particles meet the requirements of many of the applications, heterogeneous particles provide advantages in the more demanding cases. Thus, two-phase soft-hard particles have been used for coatings, which

E. Akhmatskaya

Basque Center for Applied Mathematics (BCAM),

Mazarredo 14,

48009 Bilbao, Basque Country, Spain

J. M. Asua $(\square)$

POLYMAT and Grupo de Ingeniería Química, Departamento de

Química Aplicada, Facultad de Ciencias Químicas,

University of the Basque Country UPV/EHU,

Joxe Mari Korta zentroa, Tolosa etorbidea 72,

Donostia/San Sebastián 20018, Spain

e-mail: jm.asua@ehu.es combine a low minimum film forming temperature and a high blocking resistance [4]. Rubber thermoplastic core-shell particles are useful to impart toughness to thermoplastics resins [5]. Waterborne polymer-polymer hybrids (e.g., alkyd-acrylic $[6,7]$, polyurethane-acrylic [8], and epoxy-acrylic [9]) have been developed in an attempt to combine the positive properties of both polymers, avoiding their drawbacks. Waterborne polymer-inorganic (e.g., silica and clay) hybrids led to improvements in mechanical and thermal resistances [10-14].

Because of its scientific challenge and practical importance, particle morphology has received considerable attention in literature. An excellent review [15] is available, which shows that the work is mainly restricted to twophase systems. Particle morphology depends on the interplay between thermodynamics and kinetics. The thermodynamic equilibrium morphology is the one that has minimum interfacial energy, and it depends on the interfacial tensions. For a two-phase system, the number of possible equilibrium morphologies is small (core-shell, inverted core-shell, hemispherical, and separated particles), and the equilibrium morphology is the one that gives the minimum interfacial energy calculated as the product of the interfacial areas and the interfacial tensions [16-19]. These predictions have been assessed experimentally [20-22]. For three-phase systems, the number of possible morphologies tremendously increases. Sundberg and Sundberg [23] identified 22 distinct although somehow arbitrary morphologies. To make the calculus of the interfacial area easier, some of the particle structures were a simplified sketch of the actual morphology. Even in this case, the calculation of the equilibrium morphology required tedious calculations because of the large number of morphological alternatives involved. This method cannot be applied for systems with four or more phases. A novel approach has been recently developed by Reyes and Asua [24] for the prediction of equilibrium morphologies of multiphase waterborne systems, such as polymer-polymer and polymer-polymerinorganic hybrids. 
A large number of processes are carried out under conditions in which non-equilibrium morphologies are obtained $[15,25-30]$. These morphologies are governed by kinetics. González-Ortiz and Asua developed a model of morphology development under dynamic conditions for two-phase systems [19, 31, 32]. A model for the evolution of particle morphology of two-phase systems when graft copolymer is produced in situ has been recently published [33]. However, no dynamic model for systems with three or more phases has been reported. Therefore, there is a need for models describing the kinetics of phase morphology development of multiphase systems, such as polymer-polymerinorganic composite particles. In this article, a novel model based on stochastic dynamics is developed for the prediction of the dynamics of the development of the particle morphology of multiphase waterborne systems.

\section{Mathematical model}

Complex waterborne dispersed particles are mainly produced in two-stage processes by either miniemulsion polymerization $[13,14,34-38]$ or seeded emulsion polymerization [39-41]. In miniemulsion polymerization, in the first stage, a preformed polymer is dissolved in monomer, and the mixture is dispersed in water with the help of emulsifiers. If desired, inorganic material is added during the miniemulsification process. The second stage involves the polymerization of the monomer and the development of the final morphology. In the first stage of the seeded emulsion polymerization, the seed is prepared by emulsion polymerization. In the second stage, another monomer is added and polymerized leading to the formation of the particle morphology. The inorganic material can be placed at the surface of the particle during the formation of the seed (e.g., using Pickering stabilization). This process cannot be used to place the inorganic material within the particles.

In this work, a system containing an inorganic material was used as a case study. Inorganic materials are incorporated to improve mechanical and thermal properties [11-14, 42], to provide hiding power (opacity) [43-45] and UV resistance [46] of the coatings and to stabilize latexes [47, 48]. Incorporation of quantum dots leads to a wide range of applications in areas like chemical sensors [49], optoelectronic and photonics (LED [50], solar cells [51], photonic crystals [52]), and in a large number of biomedical applications [53].

Depending on the application, it is desirable to have the inorganic particle either within the polymer particles or at the surface of the particles. In the first case, the surface of the particles should be hydrophobically modified. In the second case, some modification of the surface of the particles is still needed to render them partially hydrophobic and hence promoting their adsorption on the surface of the particles [47].
Very hydrophilic particles stay outside of the polymer particles and are not of interest in the context of this article.

Regardless of the method used in the first stage (miniemulsion vs. emulsion), the starting point of the second stage is the same for both processes: a particle containing polymer swollen with monomer and an inorganic material, which is the system simulated in this work. It was assumed that the second stage occurred in batch under isothermal conditions. In these systems, monomer mass transfer among miniemulsion particles is undesired because it leads to a broad particle composition distribution. In practice, this is minimized by using stable miniemulsions and short nucleation periods. In the simulations presented in this work, it was assumed that mass transfer through the aqueous phase was negligible.

Therefore, the particle at the beginning of the second stage was composed of a preformed polymer swollen with monomer and an inorganic material. The preformed polymer and the inorganic material will be referred to as polymer 1 and inorganic, respectively. The monomer and the polymer resulting from its polymerization will be referred to as monomer 2 and polymer 2 , respectively.

A $200-\mathrm{nm}$ particle with a composition polymer/monomer $=50 / 50 \mathrm{wt} / \mathrm{wt}$ is composed by about 1,500 polymer chains (assuming a molecular weight of $750,000 \mathrm{~g} / \mathrm{mol}$, which is very common in polymerization in dispersed media) and about $11 \times 10^{6}$ molecules of monomer. Obviously, there is no way in which such a number of monomer molecules can be treated independently. Therefore, we pulled them in packs (subparticles) in such a way that polymerization of the monomer molecules contained in one subparticle led to one polymer chain. The discretization of the monomer in subparticles does not allow a homogeneous distribution of the monomer at molecular level, but considering that the monomer contained in a particle is discretized in 1,564 subparticles, the distribution of the monomer is homogeneous if a reference volume as small as $4,000 \mathrm{~nm}^{3}$ is considered.

On the other hand, we treated the polymer chains individually (as subparticles). Assuming a density $\rho=1 \mathrm{~kg} / \mathrm{L}$ for both polymer and monomer, the diameter of each subparticle would be $\sigma=13.3 \mathrm{~nm}$. The system was assumed to contain $20 \%$ of inorganic material. In this regard, it is worth pointing out that the model can handle any level of inorganic material. On the other hand, it was considered that the system was colloidally stable. The water surrounding the particle was also pulled in subparticles. In the model, each subparticle was represented by a sphere that interacts with its neighbors. For simplicity, the inorganic and water spheres were considered to have the same size and density as the monomer and polymer spheres.

In the simulations, as a case study, the system at the beginning of the second stage was formed by polymer 1 (1,564 subparticles), monomer 2 (1,564 subparticles), and 
inorganic hybrid (782 subparticles) surrounded by water (4,330 subparticles). A spherical simulation cell of diameter $32 \sigma$ was used to fit the 8,240 subparticles of size $\sigma$. These values should be modified according to the particular system considered.

The non-bonded interactions between two subparticles $i$ and $j$ of polymer 1 -polymer 1, polymer 2-polymer 2, monomer 2-monomer 2, and water-water as well as the interaction between polymer 1 -monomer 2 and polymer $2-$ monomer 2 with relative positions $r_{i}-r_{j}=r_{i j} \hat{r}_{i j}$ (the hat indicating a unit vector) were modeled by the LennardJones potential,

$U_{\mathrm{LJ}}\left(r_{i j}\right)=4 \varepsilon_{i j}\left[\left(\frac{\sigma}{r_{i j}}\right)^{12}-\left(\frac{\sigma}{r_{i j}}\right)^{6}\right]$

where $\varepsilon_{i j}$ is the depth potential well.

A repulsive generalized soft sphere potential was used for the interactions between dissimilar phases: polymer 1-polymer 2, polymer 1-inorganic, polymer 1-water, polymer 2-inorganic, polymer 2-water, inorganic-monomer 2 , inorganic-water, and monomer 2-water. A repulsive potential was also used for inorganic-inorganic interactions because in practice the surface of the inorganic particles is modified to avoid aggregation. An incomplete modification of the surface of the inorganic particles would lead to a lower repulsion potential or even to an attractive potential.

$U_{r}\left(r_{i j}\right)=\varepsilon_{i j}\left(\frac{\sigma}{r_{i j}}\right)^{6}$

In order to keep the polymer and inorganic subparticles within the particle and to reproduce a continuous aqueous phase out of the sphere, we introduced an impenetrable structureless wall of the simulation sphere. Therefore, for polymer 1 , monomer 2 , polymer 2 , and inorganic subparticles of the system, a repulsive generalized soft sphere potential was used to prevent them from moving from the center of the simulation further than a distance $R_{\mathrm{c}}$ :

$U_{\text {poly-wall }}\left(r_{i j}\right)=\varepsilon_{i j}\left(\frac{\sigma}{r_{i j}}\right)^{6}$ for $r_{i j}>R_{\mathrm{c}}-2^{1 / 6} \sigma$

$U_{\text {poly-wall }}\left(r_{i j}\right)=0 \quad$ for $r_{i j} \leq R_{\mathrm{c}}-2^{1 / 6} \sigma$

In order to monitor the distances between subparticles and the wall, a dummy frozen particle was placed in a center of the simulation cell and used to determine the distances between the particles and the wall as a difference between the radius of the simulation cell and the distance between the particles and the dummy frozen particle.

The interactions between water spheres and the wall of the simulation cell were described in the same manner, using water-frozen dummy particle interactions, which in this case were chosen to be a Lennard-Jones potential:

$U_{\text {poly-wall }}\left(r_{i j}\right)=\varepsilon_{i j}\left(\frac{\sigma}{r_{i j}}\right)^{6}$ for $r_{i j}>R_{\mathrm{c}}-2.5 \sigma$

$U_{\text {poly-wall }}\left(r_{i j}\right)=0 \quad$ for $r_{i j} \leq R_{\mathrm{c}}-2.5 \sigma$

In Eqs. $3 \mathrm{a}, 3 \mathrm{~b}, 4 \mathrm{a}$, and $4 \mathrm{~b}, r_{i j}$ is the distance between simulation particles and the center of the simulation sphere (the frozen dummy particle), and

$R_{\mathrm{c}}=16$ for water subparticles

$R_{\mathrm{c}}=14$ for monomer and polymer subparticles

The simulation included two steps. Firstly, the structure of the monomer swollen initial particles was calculated. Secondly, the dynamics of the particle morphology development caused by polymerization of monomer 2 was simulated.

In practice, the initial waterborne dispersion is prepared by dispersing an organic phase (polymer dissolved in/swollen with monomer) in an aqueous solution of surfactant. The inorganic particles are incorporated to the organic phase when their surface is hydrophobically modified. Otherwise, they are incorporated to the aqueous phase. The dispersion is formed by using a suitable homogenization device (rotorstator, sonicator, or high-pressure homogenizer [54, 55]). Because of the presence of the monomer, the internal viscosity of the initial particles is low, and hence, after homogenization, the most likely structure is that of thermodynamic equilibrium. Therefore, the goal of the first step of the simulation was to calculate the equilibrium structure of the initial particles. In order to determine the structure of the monomer swollen initial particle, a preliminary distribution of polymer 1 , inorganic, monomer 2 , and water subparticles was chosen, and then the system was equilibrated using stochastic dynamics simulation.

The preliminary distribution of the monomer 2, polymer 1 , inorganic, and water subparticles was chosen as follows: First, the subparticles representing the polymer 1, monomer 2, inorganic, and water were positioned randomly within a simulation sphere of a diameter $D=32 \sigma$, closer to the center. The simulation sphere was surrounded by a layer of vacuum of $2.5 \sigma$. To form the proper minimized starting configuration for the equilibrating stochastic dynamics run, the steepest descent method with a dimensionless maximum step size of 0.001 and a tolerance of 10 was performed for 4,000 minimization steps for the randomly set system. The initial structure of the monomer swollen polymer particle immersed in water was then 
determined by running the stochastic dynamic simulation for $t=2,000$.

A problem associated to the random distribution of subparticles is that the density of subparticles achieved by means of the initial random placement of the subparticles in the simulation cell is lower than that at equilibrium. Therefore, rearrangement of the subparticles during the minimization of the energy of the system leads to the formation of vacuum regions that disturbs the calculation of the energy of the system because the subparticles at the borders of the vacuum regions are not subjected to interactions with other subparticles. This leads to unrealistic structures. In order to avoid this problem, first, the subparticles were randomly distributed using the values of $R_{\mathrm{c}}$ given in Eqs. $5 \mathrm{a}$ and $5 \mathrm{~b}$, and the system was equilibrated for $t=$ 2,000 . Then, the values of $R_{\mathrm{c}}$ were modified to $R_{\mathrm{c}}=15.7$ for water particles and $R_{\mathrm{c}}=13.2$ for polymer 1 , monomer 2 , and inorganic particles, and the system was equilibrated again for $t=2,000$. This gave the starting configuration for the calculation of the development of the particle morphology during polymerization using stochastic dynamics simulation. Analysis of the different initial configurations obtained with this approach showed that they were consistent with the equilibrium morphologies expected in monomer swollen inorganic-polymer initial particles formed by miniemulsification.

The dynamics of the particle morphology development was simulated by means of the velocity Langevin dynamics performed in the NVT ensemble:

$m \frac{d^{2} r}{\mathrm{~d} t^{2}}=\nabla U-\gamma m \frac{\mathrm{d} r}{\mathrm{~d} t}+\sqrt{2 \gamma k_{\mathrm{B}} T m} R(t)$

where $m$ is the mass of the subparticle, $\gamma$ is the friction factor, $k_{\mathrm{B}}$ is the Boltzmann constant, $T$ is the temperature, and

$<R(t)>=0 ;<R(t) R\left(t^{\prime}\right)>=\delta\left(t-t^{\prime}\right)$

A dimensionless Langevin equation was obtained by using $x=r / \sigma, \varepsilon^{*}=\varepsilon / \varepsilon^{\prime}, U^{*}=U / \varepsilon^{\prime}$, and $t^{*}=t / \tau$ where $\tau=$ $\sqrt{m \sigma^{2} / \varepsilon^{\prime}}$ and $\varepsilon^{\prime}$ is a reference potential that in this work was taken as $4.74 \times 10^{-21} \mathrm{~J}$.

$$
\begin{aligned}
\frac{d^{2} x}{\mathrm{~d} t *^{2}}= & \nabla U^{*}-\frac{\gamma}{\left(\frac{\varepsilon^{\prime}}{m \sigma^{2}}\right)^{0.5}} \frac{\mathrm{d} x}{\mathrm{~d} t^{*}} \\
& +\sqrt{2 \frac{\gamma}{\left(\frac{\varepsilon^{\prime}}{m \sigma^{2}}\right)^{0.5}} \frac{k_{\mathrm{B}} T}{\varepsilon^{\prime}} \frac{R^{2}(t)}{\left(\frac{\varepsilon^{\prime}}{m \sigma^{2}}\right)^{0.5}}}
\end{aligned}
$$

One of the challenges during the second stage of the synthesis of complex waterborne particles is to avoid secondary nucleation (formation of new particles by homogeneous nucleation) because the new particles formed will only contain the polymer formed from the monomer.
Homogeneous nucleation is enhanced when water-soluble initiators are used. Therefore, oil-soluble initiators are often used and were considered in most of the simulations presented in this work. Consequently, secondary nucleation was considered to be negligible.

Polymerization of monomer 2 was simulated by converting the monomer subparticles into subparticles of a new polymeric phase (polymer 2), namely a new type of subparticles appeared in the system, but the total number of simulated subparticles was constant during the simulation process. Polymerization kinetics was accounted for by means of the rate of transformation of monomer 2 subparticles into subparticles of polymer 2. For the examples presented in this article, a constant polymerization rate was used. This corresponds well to miniemulsion polymerizations using oil-soluble initiators [56]. The polymerization kinetics was simulated by considering that the conversion of monomer 2 into the polymer 2 occurred in $M$ steps. In each of these steps, $1 / M$ th of the initial monomer subparticles were randomly chosen and gradually converted into polymer 2 over a certain process period. The transformation of the monomer into the new polymer was achieved by gradually changing the values of $\varepsilon$ in the potentials from those of the monomer to the characteristic values of the new polymer. The random choice of the monomer subparticles implies a uniform distribution of radicals within the polymer particles, which is consistent with the use of oil-soluble initiators. For a polymer density of $1.1 \mathrm{~kg} / \mathrm{L}$ and a monomer density of $0.95 \mathrm{~kg} / \mathrm{L}$, this represents a reduction of $5 \%$ in size, which was neglected in the simulations.

If water-soluble initiators are used, the distribution of radicals may not be uniform because of the anchoring effect of the hydrophilic part of the entering radical to the surface of the particle. However, this is not always the case. Thus, non-charged water-soluble initiators (e.g., tertbutyl hydroperoxide that is often used in redox systems) and monomers with a high chain transfer to monomer (e.g., vinyl acetate) lead to much more homogeneous radical concentrations. In order to consider a profile of radical concentration, the distribution of radicals in the particle may be calculated using the available methods $[57,58]$ and the monomer subparticles that are going to undergo polymerization in a given time interval are chosen according to the radical concentration profile, namely giving a higher probability of reaction to the monomer subparticles that are near the surface of the particle.

The parameters of the Lennard-Jones and repulsive potentials can be estimated from the surface and interfacial tensions because methods for calculating surface and interfacial tensions from the Lennard-Jones and repulsive potentials are available [59-62]. These models show that for the Lennar-Jones potential, the fine details of dependence of the surface tension on $\varepsilon$ depend on the way the model is 
implemented, e.g., the cutoff distance of the Lennard-Jones potential, but most of the results can be approximated to

surface tension $=a \varepsilon-b k_{\mathrm{B}} T$

where $a$ and $b$ are parameters. This means that higher values of $\varepsilon$ represent higher surface tensions.

The friction factor $(\gamma)$ controls the rate at which the phases move in the particle, and it increases with the internal viscosity of the particle. High values of $\gamma$ correspond to high internal viscosity of the particle. This leads to a slow phase migration, which for fast polymerization rates it may result in a system in which polymer 2 remains in the place where it is formed. On the other hand, low values of $\gamma$ correspond to low particle viscosity that may lead to equilibrium morphologies. In addition, as the viscosity increases as $\eta \div \phi^{5}$ ( $\phi$ being the volume fraction of polymer [63]), in the simulations presented in this work, the following equation was used for the friction factor

$\gamma=\gamma_{0}(0.5+0.5 X)^{5}$

where $X$ is the conversion of monomer and $\gamma_{0}$ is the value of $\gamma$ at $X=1$. The actual value of $\gamma_{0}$ depends on the particular system considered, and for the sake of the simulations performed in this work, the value of $\gamma_{0}$ was estimated by considering that it is related to the diffusion coefficient (Df) as

$\gamma_{0}=\frac{k_{B} T}{m \mathrm{Df}}$

Polymer diffusion has been extensively investigated during film formation from latexes, and the diffusion coefficients have been estimated using fluorescence resonance energy transfer methods [64-67]. In these works, it can be seen that the value of Df depends on both the system considered and the temperature of the experiment. Most of the waterborne dispersed polymers are used to form films at ambient temperature. Therefore, the polymerization temperature is well above the $T_{\mathrm{g}}$ of the polymer. For $T=70^{\circ} \mathrm{C}$, a value of $\mathrm{Df}=5 \times 10^{-18} \mathrm{~m}^{2} \mathrm{~s}^{-1}$ is reasonable, which gives a value of $\gamma_{0}=7.6 \times 10^{17} \mathrm{~s}^{-1}$. It is worth pointing out that, under given conditions, the diffusion of a polymer chain depends on both the molecular weight and the number and length of branches (if any). As in the system there is a wide range of molecular weights and polymer architectures, Df is an average diffusion coefficient.

All simulations were performed using the GROMACS 4.0.7 code [68] in parallel on the computing cluster comprising 29 computing elements composed by two Intel QuadCore Xeon processors. The visualization molecular dynamics tool, VMD [69], has been used for analysis of the SD trajectories and for creating figures for this article. In GROMACS, the accuracy of the integration of the dimensionless Langevin equation decreases with the increasing of the product $\gamma \times \tau \times \Delta t^{*}$, where $\Delta t^{*}$ is the time increment in the integration. As a reference, $\Delta t^{*} \leq 0.005$ should often be used to integrate the dimensionless Langevin equation with a reasonable accuracy. Therefore, the corresponding real time, $\Delta t^{*}$, which is equal to $\tau \times \Delta t^{*}$, is very small (about $3 \times 10^{-11} \mathrm{~s}$ with the values of the parameters discussed above). This means that to simulate a 1 -h polymerization, $1.2 \times 10^{14}$ integration steps are needed, which results in an unaffordable computer time (the maximum affordable number of integration steps is estimated to be about $1.5 \times 10^{7}$ ).

A solution to this problem can be found considering the migration of phases in a particle containing polymer 1 , polymer 2 , and monomer 2 and in which no polymerization occurs. The movement of the phase $i$ can be described by means of a classical material balance

$$
\frac{\partial C_{i}}{\partial t}=-\nabla \cdot \operatorname{Df} \nabla C_{i}
$$

where Df is an effective diffusion coefficient that includes both Brownian and the interaction terms and $C_{i}$ is the concentration of phase $i$ (polymer 1, polymer 2, and monomer 2).

Equation 12 is interesting because if due to computation limitations it is not possible to integrate it for longer than a certain time, e.g., $1 \mathrm{~s}$, it is still possible to obtain the evolution of the concentration of phase $i$ in the real system in which the process occurs over a longer time scale, e.g., $10^{4} \mathrm{~s}$, by simply using a value of the effective diffusion coefficient equal to $\mathrm{Df} \times$ $10^{4}$. In the framework of the Langevin equation, the effective diffusion coefficient is inversely proportional to $\gamma$; therefore, if a Langevin equation is used to describe this example, it would be sufficient to use a value of $\gamma$ equal to $\gamma \times 10^{-4}$.

Therefore, in order to simulate a 1-h process using the Langevin equation with a maximum of $1.5 \times 10^{7}$ integration steps and a real-time increment of $3 \times 10^{-11} \mathrm{~s}$, the value of $\gamma_{0}$ to be used in the simulation is $\gamma_{0} /\left(8 \times 10^{6}\right)$.

For a polymerizing system, the material balance becomes:

$$
\frac{\partial C_{i}}{\partial t}=-\nabla \cdot \operatorname{Df} \nabla C_{i}+R_{i}
$$

where $R_{i}$ is the rate of generation of phase $i$ (in the case of consumption, it appears with a negative sign). From Eq. 13 it is evident that in order to simulate the system over a longer time scale, the rate of generation of phase $i$ should also be multiplied for the same factor as Df.

\section{Representative simulations}

The model was first successfully tested in the simulation of a two-phase system for which at least the equilibrium morphology is known. Then, a three-phase system composed by 


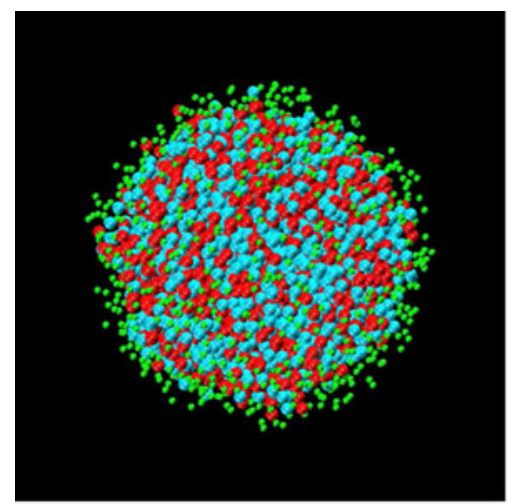

a) initial particle

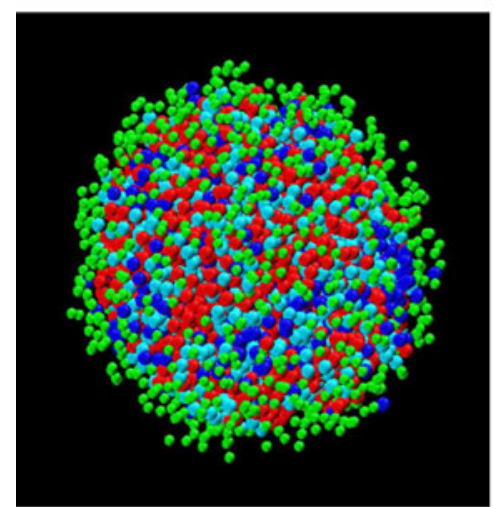

b) $\mathrm{x}=0.3 ; \mathrm{t}=1800$

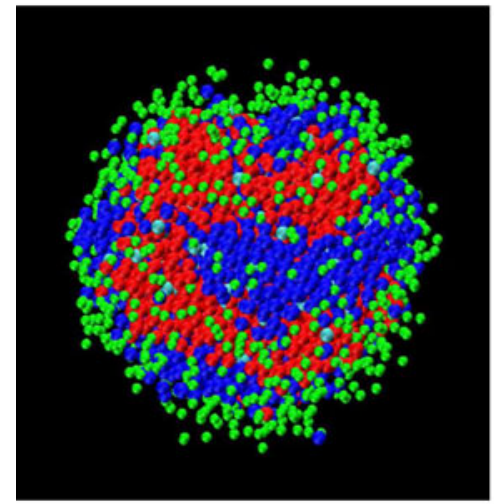

e) $\mathrm{X}=0.9 ; \mathrm{t}=5400$

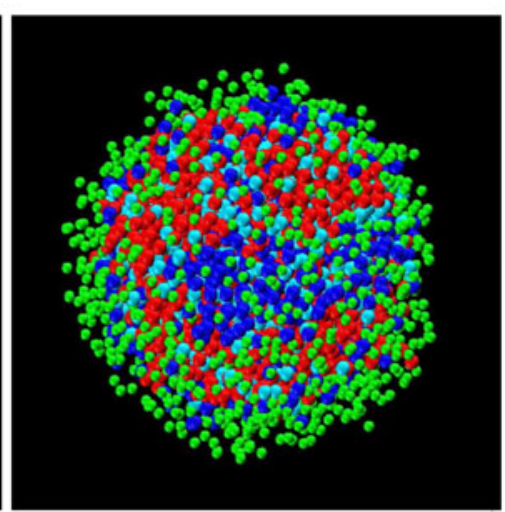

c) $\mathrm{X}=0.5 ; \mathrm{t}=3000$

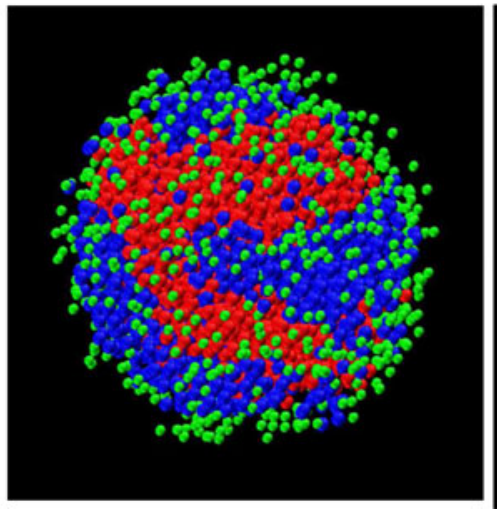

f) $X=1.0 ; t=6000$

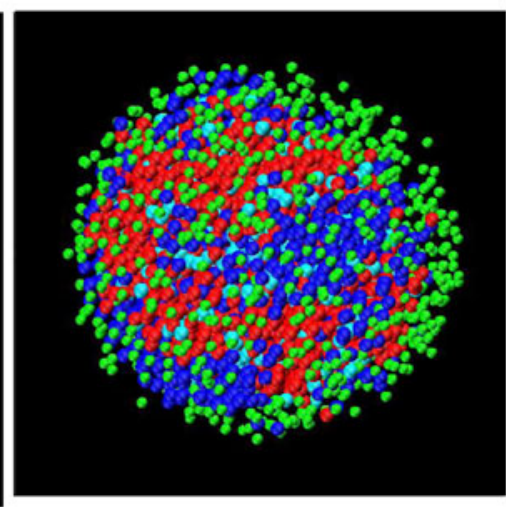

d) $X=0.7 ; t=4200$

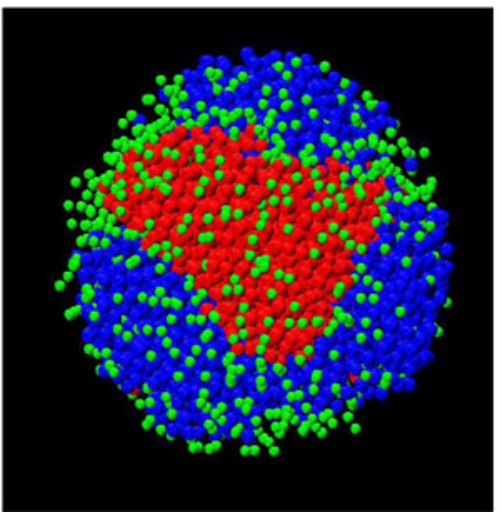

g) aging; $\mathrm{t}=15000$

Fig. 1 a-g Evolution of the particle morphology (run 1). Potential parameters are given in Table 1. Oil-soluble initiator. Polymer 1 (red); monomer 2 (cyan); polymer 2 (blue); inorganic (green); $X$ is the monomer 2 conversion; $t$ is the time in reduced units

Table 1 Values of $\varepsilon$ used in the simulations with partially hydrophobic inorganic particles (runs 1-3)

\begin{tabular}{|c|c|c|c|c|c|c|}
\hline & Polymer 1 & Monomer 2 & Polymer 2 & Inorganic & Water & Wall \\
\hline Polymer 1 & $\varepsilon_{\mathrm{P} 1-\mathrm{P} 1}=1.3$ & $\varepsilon_{\mathrm{P} 1-\mathrm{M} 2}=1.3$ & $\varepsilon_{\mathrm{P} 1-\mathrm{P} 2}=0.1$ & $\varepsilon_{\mathrm{P} 1-\mathrm{Inor}}=0.2$ & $\varepsilon_{\mathrm{P} 1-\mathrm{W}}=3$ & $\varepsilon_{\mathrm{P} 1-\text { wall }}=6$ \\
\hline Monomer 2 & $\varepsilon_{\mathrm{M} 2-\mathrm{P} 1}=1.3$ & $\varepsilon_{\mathrm{M} 2-\mathrm{M} 2}=1.3$ & $\varepsilon_{\mathrm{M} 2-\mathrm{P} 2}=1.3$ & $\varepsilon_{\mathrm{M} 2-\mathrm{inor}}=0.1$ & $\varepsilon_{\mathrm{M} 2-\mathrm{W}}=3$ & $\varepsilon_{\mathrm{M} 2-\mathrm{wall}}=6$ \\
\hline Polymer 2 & $\varepsilon_{\mathrm{P} 2-\mathrm{P} 1}=0.1$ & $\varepsilon_{\mathrm{P} 2-\mathrm{M} 2}=1.3$ & $\varepsilon_{\mathrm{P} 2-\mathrm{P} 2}=1.3$ & $\varepsilon_{\mathrm{P} 2-\mathrm{inor}}=0.1$ & $\varepsilon_{\mathrm{P} 2-\mathrm{W}}=1$ & $\varepsilon_{\mathrm{P} 2-\mathrm{wall}}=6$ \\
\hline Inorganic & $\varepsilon_{\text {inor-P1 }}=0.2$ & $\varepsilon_{\text {inor-M2 }}=0.1$ & $\varepsilon_{\text {inor-P2 }}=0.1$ & $\varepsilon_{\text {inor-inor }}=0.1$ & $\varepsilon_{\text {inor-W }}=0.3$ & $\varepsilon_{\text {inor-wall }}=6$ \\
\hline Water & $\varepsilon_{\mathrm{W}-\mathrm{P} 1}=3$ & $\varepsilon_{\mathrm{W}-\mathrm{M} 2}=3$ & $\varepsilon_{\mathrm{W}-\mathrm{P} 2}=1$ & $\varepsilon_{\mathrm{W}-\mathrm{inor}}=0.3$ & $\varepsilon_{\mathrm{W}-\mathrm{W}}=1$ & $\varepsilon_{\mathrm{W}-\mathrm{wall}}=1$ \\
\hline Wall & $\varepsilon_{\mathrm{wall-P} 1}=6$ & $\varepsilon_{\mathrm{wall-M} 2}=6$ & $\varepsilon_{\mathrm{wall-P} 2}=6$ & $\varepsilon_{\text {wall-inor }}=6$ & $\varepsilon_{\text {wall-W }}=1$ & \\
\hline
\end{tabular}




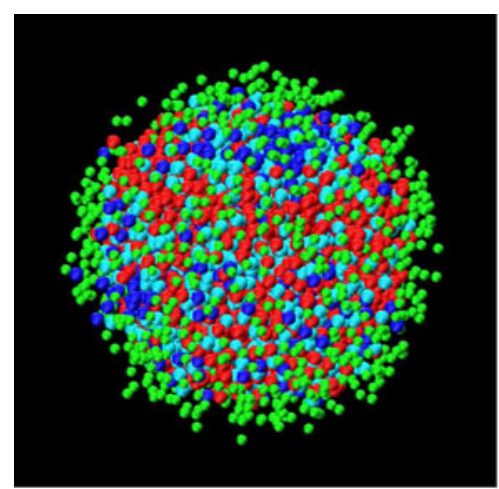

a) $X=0.3 ; t=6000$

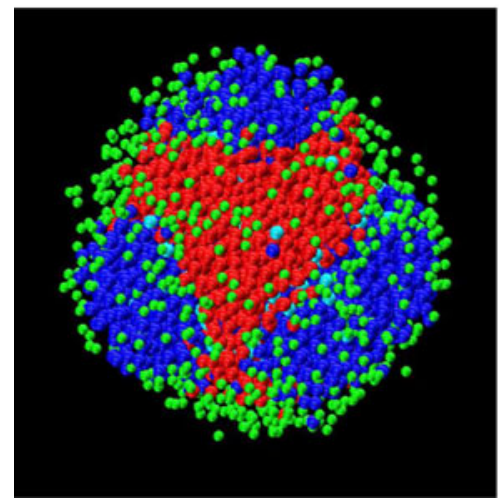

d) $X=0.9 ; t=18000$

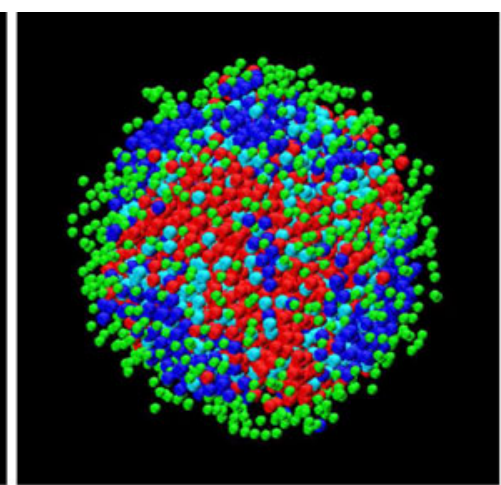

b) $X=0.5 ; t=10000$

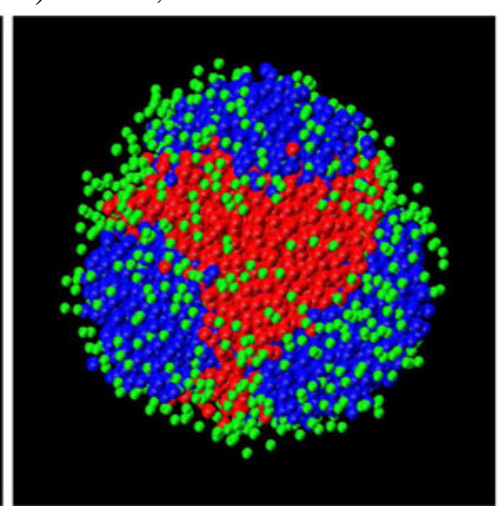

e) $X=1.0 ; t=20000$

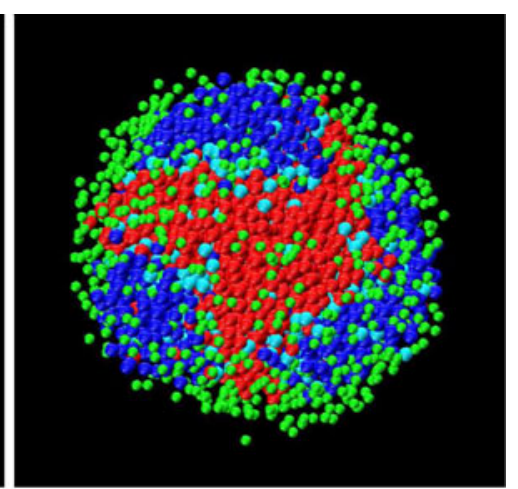

c) $X=0.7 ; t=14000$

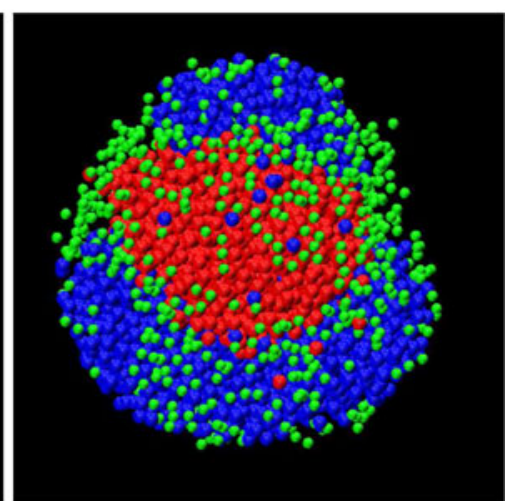

f) aging; $t=48000$

Fig. 2 a-f Particle morphology evolution: slower polymerization rate (run 2). Potential parameters are given in Table 1. Oil-soluble initiator. Polymer 1 (red); monomer 2 (cyan); polymer 2 (blue); inorganic (green); $X$ is the monomer 2 conversion; $t$ is the time in reduced units

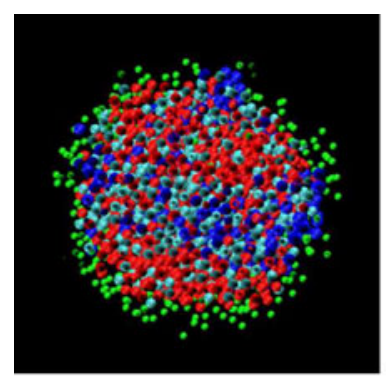

Oil-soluble init

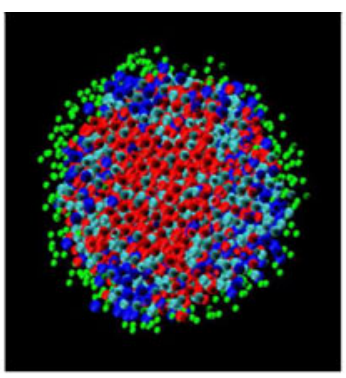

water-soluble init

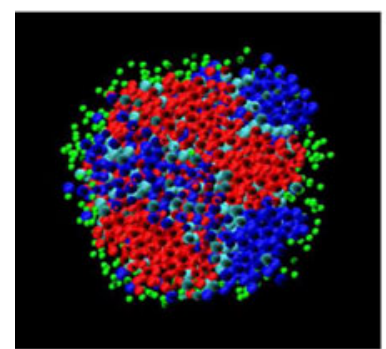

Oil-soluble init

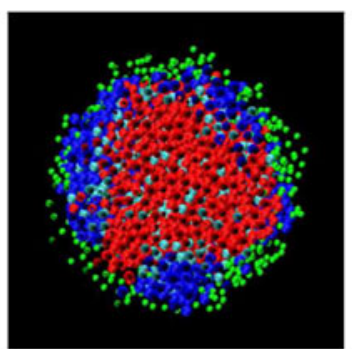

water-soluble init

$$
\text { c) } \mathrm{X}=0.7 ; \mathrm{t}=6000
$$

Fig. 3 a-d Effect of the type of initiator on particle morphology evolution: slower polymerization rate (run 2: oil-soluble initiator; run 3: water-soluble initiator). Potential parameters are given in Table 1.

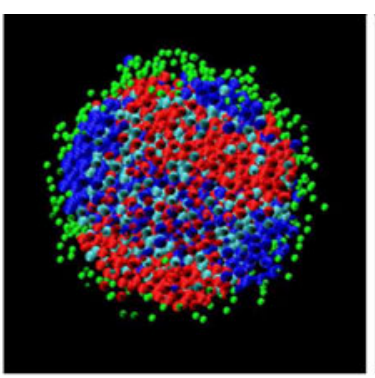

Oil-soluble init

b) $\mathrm{X}=0.5 ; \mathrm{t}=10000$

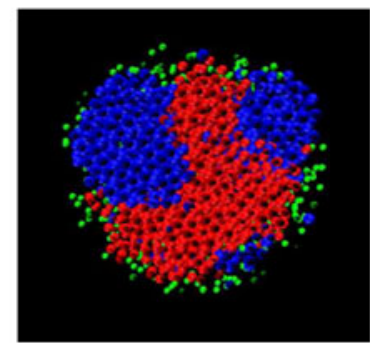

Oil-soluble init

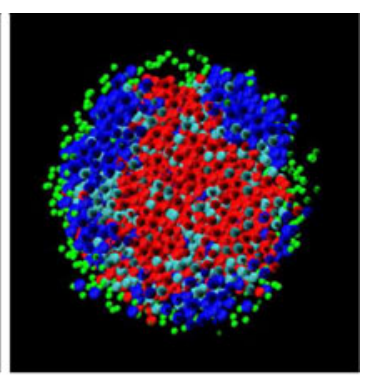

water-soluble init

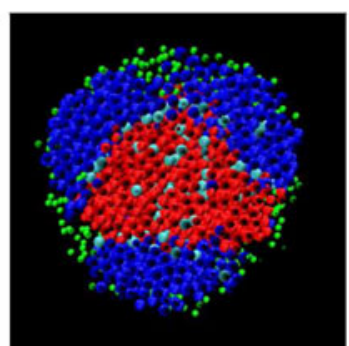

water-soluble init d) $X=1.0 ; t=20000$

Polymer 1 (red); monomer 2 (cyan); polymer 2 (blue); inorganic (green); $X$ is the monomer 2 conversion; $t$ is the time in reduced units 


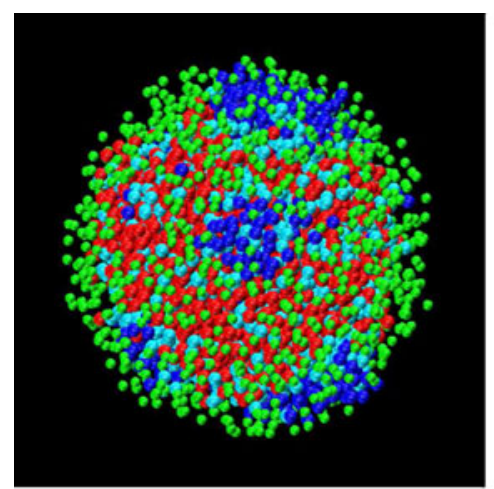

a) $X=0.3 ; t=6000$

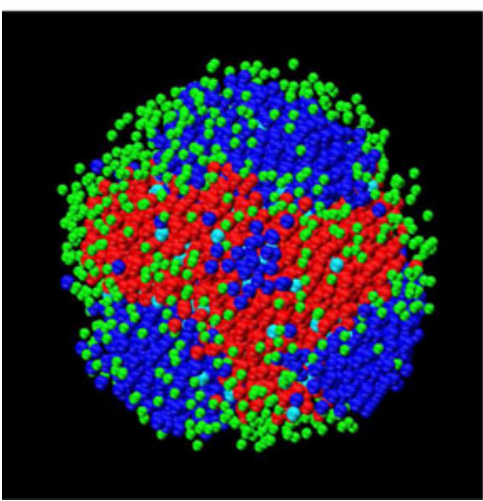

d) $X=0.9 ; t=18000$

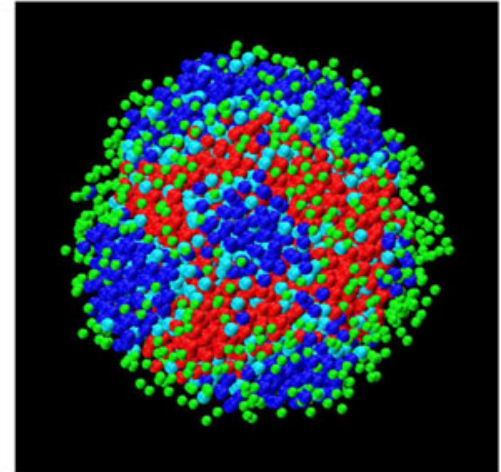

b) $X=0.5 ; t=10000$

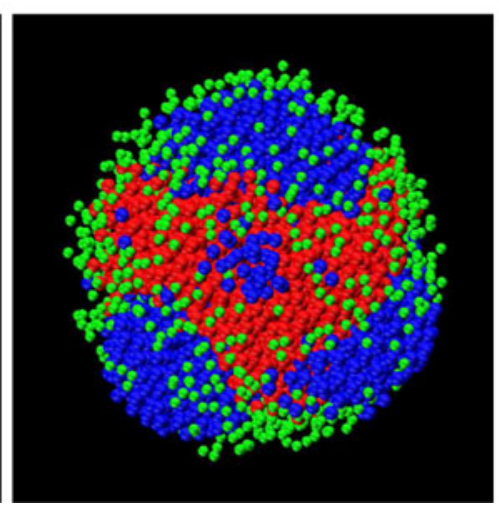

e) $X=1.0 ; t=20000$

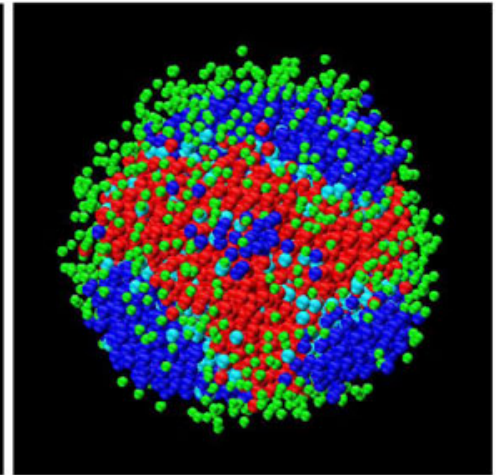

c) $X=0.7 ; t=14000$

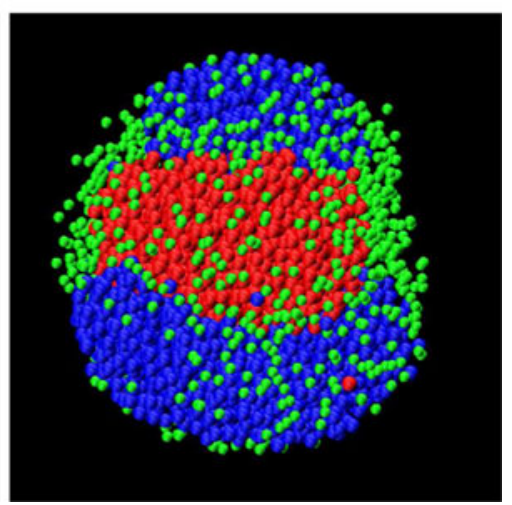

f) aging; $t=48000$

Fig. 4 a-f Particle morphology evolution: slow polymerization rate (run 4). Potential parameters are given in Table 2. Oil-soluble initiator. Polymer 1 (red); monomer 2 (cyan); polymer 2 (blue); inorganic (green); $X$ is the monomer 2 conversion; $t$ is the time in reduced units

polymer 1 (1,564 subparticles), monomer2/polymer 2 (1,564 subparticles), and inorganic material (782 subparticles) surrounded by water (4,330 subparticles) was considered. The initial particle contained polymer 1 , monomer 2 , and the inorganic material.

First, the case of partially hydrophilic inorganic particles with polymer 2 more hydrophilic than polymer 1 was considered. Figure 1 presents the evolution of the particle morphology in the first experiment, run 1, simulated considering an oil-soluble initiator and using the parameters given in Table 1. Figure 1a shows the morphology of the initial particle. Monomer 2 and polymer 1 were uniformly distributed in the particle, which corresponds to a system in which polymer 1 is soluble in monomer 2 . The inorganic particles were placed at the surface of the particle. This system represents well the initial state of a miniemulsion polymerization aiming at producing a polymer-polymerinorganic hybrid.

Polymerization of monomer 2 (cyan) led to the formation of polymer 2 (blue), and this polymer was not compatible with polymer 1 (red). Therefore, phase separation occurred and the more hydrophobic polymer (polymer 1) accumulated in the core of the particle. It is worth pointing out that, in the context of this work, compatibility refers to interfacial tension and phase separation refers to the movement of the phases to form domains. The higher the interfacial tension
Table 2 Values of $\varepsilon$ used in the simulation of run 4

\begin{tabular}{|c|c|c|c|c|c|c|}
\hline & Polymer 1 & Monomer 2 & Polymer 2 & Inorganic & Water & Wall \\
\hline Polymer 1 & $\varepsilon_{\mathrm{P} 1-\mathrm{P} 1}=1.3$ & $\varepsilon_{\mathrm{P} 1-\mathrm{M} 2}=1.3$ & $\varepsilon_{\mathrm{P} 1-\mathrm{P} 2}=0.4$ & $\varepsilon_{\mathrm{P} 1-\mathrm{Inor}}=0.3$ & $\varepsilon_{\mathrm{P} 1-\mathrm{W}}=3$ & $\varepsilon_{\mathrm{P} 1-\text { wall }}=6$ \\
\hline Monomer 2 & $\varepsilon_{\mathrm{M} 2-\mathrm{P} 1}=1.3$ & $\varepsilon_{\mathrm{M} 2-\mathrm{M} 2}=1.3$ & $\varepsilon_{\mathrm{M} 2-\mathrm{P} 2}=1.3$ & $\varepsilon_{\mathrm{M} 2-\mathrm{inor}}=0.3$ & $\varepsilon_{\mathrm{M} 2-\mathrm{W}}=3$ & $\varepsilon_{\mathrm{M} 2-\mathrm{wall}}=6$ \\
\hline Polymer 2 & $\varepsilon_{\mathrm{P} 2-\mathrm{P} 1}=0.4$ & $\varepsilon_{\mathrm{P} 2-\mathrm{M} 2}=1.3$ & $\varepsilon_{\mathrm{P} 2-\mathrm{P} 2}=1.3$ & $\varepsilon_{\mathrm{P} 2-\mathrm{inor}}=0.3$ & $\varepsilon_{\mathrm{P} 2-\mathrm{W}}=2$ & $\varepsilon_{\mathrm{P} 2-\mathrm{wall}}=6$ \\
\hline Inorganic & $\varepsilon_{\text {inor-P1 }}=0.3$ & $\varepsilon_{\text {inor-M2} 2}=0.3$ & $\varepsilon_{\text {inor-P2 }}=0.3$ & $\varepsilon_{\text {inor-inor }}=0.1$ & $\varepsilon_{\text {inor-W }}=0.3$ & $\varepsilon_{\text {inor-wall }}=6$ \\
\hline Water & $\varepsilon_{\mathrm{W}-\mathrm{P} 1}=3$ & $\varepsilon_{\mathrm{W}-\mathrm{M} 2}=3$ & $\varepsilon_{\mathrm{W}-\mathrm{P} 2}=2$ & $\varepsilon_{\mathrm{W}-\mathrm{inor}}=0.3$ & $\varepsilon_{\mathrm{W}-\mathrm{W}}=1$ & $\varepsilon_{\mathrm{W}-\mathrm{wall}}=1$ \\
\hline Wall & $\varepsilon_{\mathrm{wall-P} 1}=6$ & $\varepsilon_{\mathrm{wall-M} 2}=6$ & $\varepsilon_{\mathrm{wall-P} 2}=6$ & $\varepsilon_{\text {wall-inor }}=6$ & $\varepsilon_{\text {wall-W }}=1$ & \\
\hline
\end{tabular}




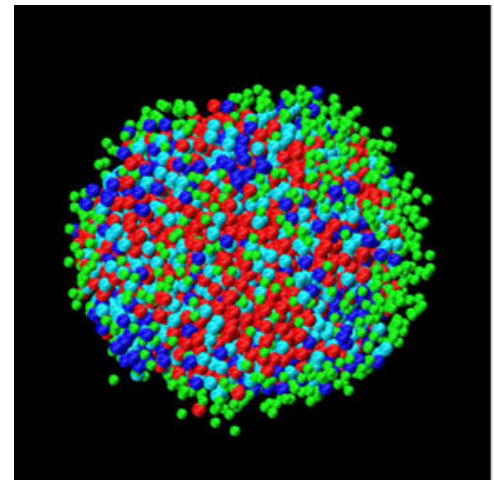

a) $X=0.3 ; t=6000$

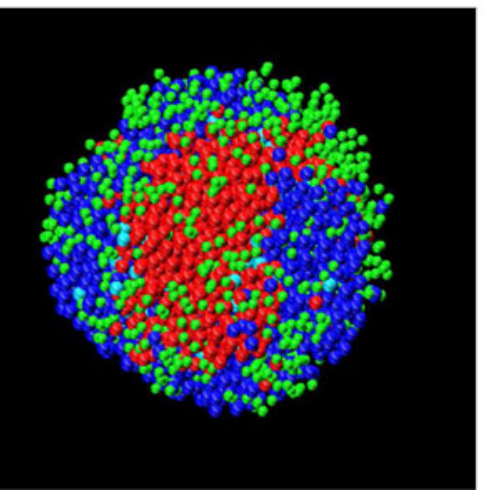

d) $X=0.9 ; t=18000$

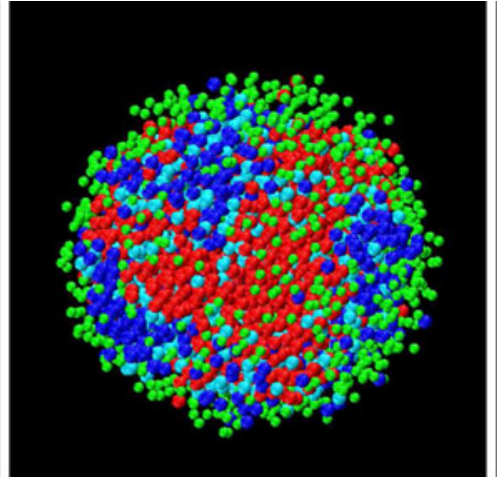

b) $X=0.5 ; t=10000$

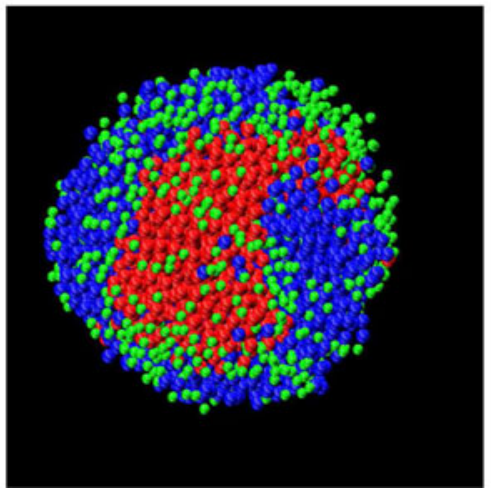

e) $\mathrm{X}=1.0 ; \mathrm{t}=20000$

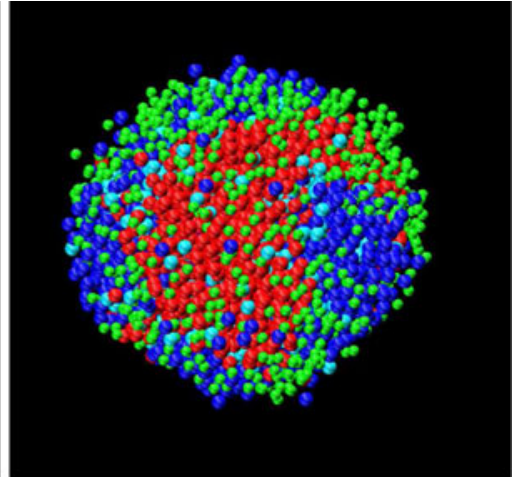

c) $X=0.7 ; t=14000$

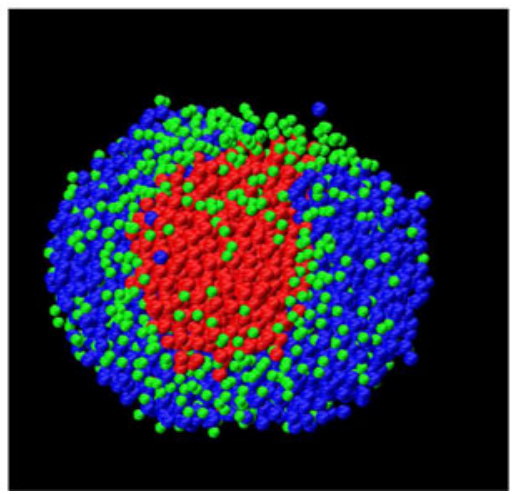

f) aging; $t=48000$

Fig. 5 a-f Particle morphology evolution: slow polymerization rate (run 5). Potential parameters are given in Table 3. Oil-soluble initiator. Polymer 1 (red); monomer 2 (cyan); polymer 2 (blue); inorganic (green); $X$ is the monomer 2 conversion; $t$ is the time in reduced units

(i.e., the higher the value of $\varepsilon$ ), the less compatible the phases. At the end of the polymerization (Fig. 1f), the particle presented a non-equilibrium morphology. Because of the high internal viscosity, particle morphology remained almost unchanged upon aging (Fig. 1g).

Figure 2 presents the evolution of the particle morphology in the case of a slower polymerization rate (run 2). Comparison with Fig. 1 shows that for a given conversion, the morphology of the particle was closer to the equilibrium morphology than in run 1 . The reason is that the polymer had more time to migrate toward the equilibrium morphology. However, even after aging, because of the high internal viscosity, the final morphology was still not at equilibrium.

Runs 1 and 2 were simulated for an oil-soluble initiator. The effect of using a water-soluble initiator that leads to a radical concentration profile was simulated for the case of a slow polymerization (as the one used in run 2) using the parameters given in Table 1. The radical concentration profile was characterized by the relative probability for reaction used in these simulations

Prob $=0.2+0.2 x+0.6 x^{2}$

where $x=r / \sigma$. Equation 14 determines that polymerization of monomer 2 located near the surface of the particle was more likely than that in the center of the particle.

Figure 3 compares the cross sections obtained in this simulation with those from run 2 (simulated using an oilsoluble initiator and the same polymerization rate and parameters; Table 1). It can be seen that when a water-
Table 3 Values of $\varepsilon$ used in the simulations with hydrophobic inorganic particles (run 5)

\begin{tabular}{|c|c|c|c|c|c|c|}
\hline & Polymer 1 & Monomer 2 & Polymer 2 & Inorganic & Water & Wall \\
\hline Polymer 1 & $\varepsilon_{\mathrm{P} 1-\mathrm{P} 1}=1.3$ & $\varepsilon_{\mathrm{P} 1-\mathrm{M} 2}=1.3$ & $\varepsilon_{\mathrm{P} 1-\mathrm{P} 2}=0.1$ & $\varepsilon_{\mathrm{P} 1-\mathrm{Inor}}=0.2$ & $\varepsilon_{\mathrm{P} 1-\mathrm{W}}=3$ & $\varepsilon_{\mathrm{P} 1-\mathrm{wall}}=6$ \\
\hline Monomer 2 & $\varepsilon_{\mathrm{M} 2-\mathrm{P} 1}=1.3$ & $\varepsilon_{\mathrm{M} 2-\mathrm{M} 2}=1.3$ & $\varepsilon_{\mathrm{M} 2-\mathrm{P} 2}=1.3$ & $\varepsilon_{\mathrm{M} 2 \text {-inor }}=0.1$ & $\varepsilon_{\mathrm{M} 2-\mathrm{W}}=3$ & $\varepsilon_{\mathrm{M} 2-\mathrm{wall}}=6$ \\
\hline Polymer 2 & $\varepsilon_{\mathrm{P} 2-\mathrm{P} 1}=0.1$ & $\varepsilon_{\mathrm{P} 2-\mathrm{M} 2}=1.3$ & $\varepsilon_{\mathrm{P} 2-\mathrm{P} 2}=1.3$ & $\varepsilon_{\mathrm{P} 2-\text { inor }}=0.1$ & $\varepsilon_{\mathrm{P} 2-\mathrm{W}}=1$ & $\varepsilon_{\mathrm{P} 2-\text { wall }}=6$ \\
\hline Inorganic & $\varepsilon_{\text {inor-P1 } 1}=0.2$ & $\varepsilon_{\text {inor-M2 }}=0.1$ & $\varepsilon_{\text {inor-P2 }}=0.1$ & $\varepsilon_{\text {inor-inor }}=0.1$ & $\varepsilon_{\text {inor-W }}=3$ & $\varepsilon_{\text {inor-wall }}=6$ \\
\hline Water & $\varepsilon_{\mathrm{W}-\mathrm{P} 1}=3$ & $\varepsilon_{\mathrm{W}-\mathrm{M} 2}=3$ & $\varepsilon_{\mathrm{W}-\mathrm{P} 2}=1$ & $\varepsilon_{\mathrm{W}-\mathrm{inor}}=3$ & $\varepsilon_{\mathrm{W}-\mathrm{W}}=1$ & $\varepsilon_{\mathrm{W}-\mathrm{wall}}=1$ \\
\hline Wall & $\varepsilon_{\text {wall-P1 }}=6$ & $\varepsilon_{\mathrm{wall-M} 2}=6$ & $\varepsilon_{\mathrm{wall-P} 2}=6$ & $\varepsilon_{\text {wall-inor }}=6$ & $\varepsilon_{\mathrm{wall-W}}=1$ & \\
\hline
\end{tabular}


soluble initiator was used, polymer 2 accumulates in the region in which it is formed, namely near the surface of the particle.

Figure 4 shows the evolution of the particle morphology in run 4 that corresponds to a system in which partially hydrophilic inorganic particles were used, but polymer 2 was more hydrophobic than in runs 1-3. In addition, the compatibilities between the polymers and between the polymers and the inorganic material were reduced. A slow polymerization rate (as in run 2) was used. Table 2 presents the values of $\varepsilon$ used in run 4 . Figure 4 shows that particle morphology evolved toward a kind of hemispherical morphology with the inorganic material partially covering the polymer phases. Nevertheless, even upon aging, the equilibrium morphology was not reached.

Figure 5 presents the evolution of the particle morphology in a system that contained inorganic particles as hydrophobic as polymer 1 (run 5). The values of $\varepsilon$ used in the simulation are given in Table 3. In this system, polymer 1 was more compatible with polymer 2 than with the inorganic particles. An oil-soluble initiator with a slow polymerization rate was used. It can be seen that in the initial particle, the inorganic material was at the outer part of the particle. The reason was that the inorganic material was incompatible with both monomer 2 and polymer 1. A strong phase separation occurred during polymerization, and when $100 \%$ conversion was reached, the inorganic particles were partially covered by the more hydrophilic polymer 2. However, even after aging, the equilibrium morphology was not reached.

\section{Conclusions}

In this work, a dynamic model was developed for the prediction of the evolution of the particle morphology of multiphase waterborne systems. The model accounts for the effects of phase compatibility and internal viscosity of the particles and is able to predict the morphologies of interesting new materials such as polymer-polymer and polymerpolymer-inorganic complex hybrids, which could not be calculated with the existing methods.

Acknowledgments The financial support from the Diputacion Foral de Gipuzkoa, Basque Government (GV IT373-10 and Saiotek 2010SPE 10UN25) and Ministerio de Ciencia y Tecnologia (CTQ 201125572) as well as the use of the computing facilities of I2BASQUE are gratefully acknowledged.

\section{References}

1. Asua JM (ed) (1997) Polymeric dispersions: principles and applications. Kluwer Academic, Dordrecht
2. Lovell PA, El-Aasser MS (eds) (1997) Emulsion polymerization and emulsion polymers. Wiley, Chichester

3. Urban D, Takamura K (2002) Polymer dispersions and their industrial applications. Wiley- $\mathrm{VCH}$, Weinheim

4. Schuler B, Baumstark R, Kirsch S, Pfau A, Sandor M, Zosel A (2000) Structure and properties of multiphase particles and their impact on the performance of architectural coatings. Prog Org Coat 40:139-150

5. Sato F, Tateyama M (1988) Impact resistant methacrylic resin composition. US 47300023

6. Tsavalas JG, Schork FJ, Landfester K (2004) Particle morphology development in hybrid miniemulsion polymerization. J Coat Technol Res 1:53-63

7. Goikoetxea M, Minari RJ, Beristain I, Paulis M, Barandiaran MJ, Asua JM (2009) Polymerization kinetics and microstructure of waterborne acrylic/alkyd nanocomposites synthesized by miniemulsion. J Polym Sci Part A Polym Chem 47:4871-4885

8. Li CY, Chiu WY, Don TM (2007) Morphology of PU/PMMA hybrid particles from miniemulsion polymerization: thermodynamic considerations. J Polym Sci Part A Polym Chem 45:3359-3369

9. Kawahara H, Goto T, Ohnishi K, Ogura H, Kage H, Matsuno Y (2001) Preparation of epoxy resin/acrylic composite latexes by miniemulsion polymerization method. J Appl Polym Sci 81:128-133

10. Bourgeat-Lami E (2002) Organic-inorganic nanostructured colloids. J Nanosci Nanotechnol 2:1-24

11. Negrete-Herrera N, Putaux JL, Laurent D, Bourgeat-Lami E (2006) Polymer/laponite composite colloids through emulsion polymerization: influence of the clay modification level on particle morphology. Macromolecules 39:9177-9184

12. Cheng J, Chen M, Zhou S, Wu L (2006) Preparation of $\mathrm{SiO}_{2} /$ PMMA composite particles via conventional emulsion polymerization. J Polym Sci Part A Polym Chem 44:3807-3816

13. Diaconu G, Asua JM, Paulis M, Leiza JR (2007) High-solids content waterborne polymer-clay nanocomposites. Macromol Symp 259:305-317

14. Diaconu G, Micusik M, Bonnefond A, Paulis M, Leiza JR (2009) Macroinitiator and macromonomer modified montmorillonite for the synthesis of acrylic/MMT nanocomposite latexes. Macromolecules 42:3316-3325

15. Sundberg DC, Durant YG (2003) Latex particle morphology, fundamental aspects: a review. Polym React Eng 11:379-432

16. Torza S, Mason SG (1970) Three-phase interactions in shear and electrical fields. J Colloid Interface Sci 33:67-83

17. Winzor CL, Sundberg DC (1992) Conversion dependent morphology predictions for composite emulsion polymers: 1 . Synthetic lattices. Polymer 33:3797-3810

18. Chen YC, Dimonie V, El-Aasser MS (1991) Effect of interfacial phenomena on the development of particle morphology in a polymer latex system. Macromolecules 24:3779-3787

19. González-Ortiz LJ, Asua JM (1995) Development of particle morphology in emulsion polymerization. 1. Cluster dynamics. Macromolecules 28:3135-3145

20. Shen S, El-Aasser MS, Dimonie VL, Vanderhoff JW, Sudol ED (1991) Preparation and morphological characterization of microscopic composite particles. J Polym Sci Part A Polym Chem 29:857-867

21. Kirsch S, Pfau A, Stubbs J, Sundberg DC (2001) Control of particle morphology and film structures of carboxylated poly(n butylacrylate/poly(methyl methacrylate) composite latex particles. Colloid Surf A Phys Eng Asp 183-185:725-737

22. Colombini D, Ljungberg N, Hassander H, Karlsson O (2005) The effect of polymerisation route on the amount of interphase in structured latex particles and their corresponding films. Polymer 46:1295-1308

23. Sundberg E, Sundberg DC (1993) Morphology development for three component emulsion polymers - theory and experiments. J Appl Polym Sci 47:1277-1294 
24. Reyes Y, Asua JM (2010) Modeling multiphase latex particle equilibrium morphology. J Polym Sci Part A Polym Chem 48:2579-2583

25. Stubbs J, Durant YG, Sundberg DC (2003) Polymer phase separation in composite latex particles. 1. Considerations for the nucleation and growth mechanism. C R Chem 6:1217-1232

26. Stubbs J, Karlsson O, Jönsson JE, Sundberg E, Durant Y, Sundberg DC (1999) Non-equilibrium particle morphology development in seeded emulsion polymerization. 1: penetration of monomer and radicals as a function of monomer feed rate during second stage polymerization. Colloid Surf A Phys Eng Aspects 153:255-270

27. Stubbs J, Carrier R, Sundberg DC (2008) Monte Carlo simulation of emulsion polymerization kinetics and the evolution of latex particle morphology and polymer chain architecture. Macromol Theory Simul 17:147-162

28. Lee CF (2005) Effects of surfactants on the morphology of composite polymer particles produced by two-stage seeded emulsion polymerization. J Polym Sci Part A Polym Chem 43:2224-2236

29. Tillier DL, Meuldijk J, Höhne GWH, Frederik PM, Regev O, Koning CO (2005) About morphology in ethylene-propylene (-diene) copolymers-based latexes. Polymer 46:7094-7108

30. Stubbs J, Sundberg DC (2008) The dynamics of morphology development in multiphase latex particles. Prog Org Coat 61:156-165

31. González-Ortiz LJ, Asua JM (1996) Development of particle morphology in emulsion polymerization. 2. Cluster dynamics in reacting systems. Macromolecules 29:383-389

32. González-Ortiz LJ, Asua JM (1996) Development of particle morphology in emulsion polymerization. 2. Cluster nucleation and dynamics in polymerizing systems. Macromolecules 29:4520-4527

33. Akhmatskaya E, Asua JM (2012) Dynamic modeling of the morphology of latex particles with in situ formation of graft copolymer. J Polym Sci Part A Polym Chem 50:1383-1393

34. Landfester K, Rothe R, Antonietti M (2002) Convenient synthesis of fluorinated latexes and core-shell structures by miniemulsion polymerization. Macromolecules 35:1658-1662

35. Li M, Daniels ES, Dimonie V, Sudol ED, El-Aasser MS (2005) Preparation of polyurethane/acrylic hybrid nanoparticles via a miniemulsion polymerization process. Macromolecules 38:4183-4192

36. Boutti S, Bourgeat-Lami E, Zydowicz N (2005) Silica/polyamide nanocomposite synthesis via an original double emulsification process in miniemulsion. Macromol Rapid Commun 26:1860-1865

37. Rodriguez R, de las Heras Alarcon C, Ekanayake P, McDonald PJ, Keddie JL, Barandiaran MJ, Asua JM (2008) Correlation of silicone incorporation with the hydrophobic and thermal properties of hybrid silicone-acrylic coatings. Macromolecules 41:8537-8546

38. Wang T, de las Heras Alarcon C, Goikoetxea M, Beristain I, Paulis M, Barandiaran MJ, Asua JM, Keddie JL (2010) Crosslinked network development in compatibilized alkyd/acrylic hybrid latex films for the creation of hard coatings. Langmuir 26:14323-14333

39. Jönsson JEL, Hassander H, Törnell B (1994) Polymerization conditions and the development of a core-shell morphology in PMMA/PS latex particles. 1. Influence of initiator properties and mode of monomer addition. Macromolecules 27:1932-1937

40. Rajatapiti P, Dimonie VL, El-Aasser MS (1995) In-situ synthesis of PBA-graft-PMMA copolymers to be used as compatibilizing agents in PBA/PMMA composite latex particles via the macromonomer method. J Macromol Sci Pure Appl Chem A32:14451460

41. Herrera V, Pirri R, Leiza JR, Asua JM (2006) Effect of in-situ formation of block copolymers on the latex particle morphology. Macromolecules 39:6969-6974

42. Bourgeat-Lami E, Espiard P, Guyot A, Gauthier C, David L, Vigier G (1996) Emulsion polymerization in the presence of colloidal silica particles. Angew Makromol Chem 242:105-122
43. Erdem B, Sudol ED, Dimonie VL, El-Aasser MS (2000) Encapsulation of inorganic particles via miniemulsion polymerization(I). Dispersion of titanium dioxide particles in organic media using OLOA 370 as stabilizer. J Polym Sci Part A Polym Chem 38:4419-4430

44. Erdem B, Sudol ED, Dimonie VL, El-Aasser MS (2000) Encapsulation of inorganic particles via miniemulsion polymerization (II). Preparation and characterization of styrene miniemulsion droplets containing $\mathrm{TiO}_{2}$ particles. J Polym Sci Part A Polym Chem 38:44314440

45. Erdem B, Sudol ED, Dimonie VL, El-Aasser MS (2000) Encapsulation of inorganic particles via miniemulsion polymerization(III). Characterization of encapsulation. J Polym Sci Part A Polym Chem 38:4441-4450

46. Pattamasattayasonthi N, Chaochanchaikul K, Rosarpitak V, Sombatsompop N (2011) Effects of UV weathering and a $\mathrm{CeO}_{2}$ based coating layer on the mechanical and structural changes of wood/PVC composites. J Vinyl Addit Technol 17:9-16

47. Binks BP (2002) Particles as surfactants - similarities and differences. Curr Opin Colloid Interf Sci 7:21-41

48. Cauvin S, Colver PJ, Bon SAF (2005) Pickering stabilized miniemulsion polymerization: preparation of clay armored latexes. Macromolecules 38:7887-7889

49. Galian RE, Guardia M (2009) The use of quantum dots in organic chemistry. Trends Anal Chem 394:47-59

50. Mattoussi H, Radzilowski LH, Dabbousi BO, Thomas EL, Bawendi MG, Rubner MF (1998) Electroluminescence from heterostructures of poly(phenylene vinylene) and inorganic CdSe nanocrystals. J Appl Phys 83:7965-7974

51. Gu I, Fromer NA, Geier ML, Alivisatos AP (2005) Air-stable all-inorganic nanocrystal solar cells processed from solution. Science 310:462-465

52. Woggon U, Wannemacher R, Artemyev MV, Moller B, Le Thomas N, Anikeyev V, Schöps O (2003) Dot-in-a-dot: electronic and photonic confinement in all three dimensions. Appl Phys B 77:469-484

53. Bruchez M, Moronne M, Gin P, Weiss S, Alivisatos AP (1998) Semiconductor nanocrystals as fluorescent biological labels. Science 281:2013-2016

54. Manea M, Chemtob A, Paulis M, de la Cal JC, Barandiaran MJ, Asua JM (2008) Miniemulsification in high pressure homogenizers. AIChEJ 54:289-297

55. Lopez A, Chemtob A, Milton JL, Manea M, Paulis M, Barandiaran MJ, Theisinger S, Landfester K, Hergeth WD, Udagama R, McKenna T, Simal F, Asua JM (2008) Miniemulsification of monomer-resin hybrid systems. Ind Eng Chem Res 47:6289-6297

56. Alduncin JA, Asua JM (1994) Molecular-weight distributions in the miniemulsion polymerization of styrene initiated by oil-soluble initiators. Polymer 35:3758-3765

57. Chern CS, Poehlein GW (1987) Polymerization in nonuniform latex particles: distribution of free radicals. J Polym Sci Part Polym Chem Ed 25:617-635

58. de la Cal JC, Urzay R, Zamora A, Forcada J, Asua JM (1990) Simulation of the latex particle morphology. J Polym Sci Part A Polym Chem 28:1011-1031

59. Binder K (1982) Monte Carlo calculation of the surface tension for two- and three-dimensional lattice-gas models. Phys Rev A 25:1699-1709

60. Salomons E, Mareschal M (1991) Surface tension, adsorption and surface entropy of liquid-vapour by atomistic simulation. J Phys Condens Matter 3:3645-3661

61. Singh JK, Kofke DA, Errington JR (2003) Surface tension and vapor-liquid phase coexistence of the square-well fluid. J Chem Phys 119:3405-3412

62. Gloor GJ, Jackson G, Blas FJ, de Miguel E (2005) Test-area simulation method for the direct determination of the interfacial 
tension of systems with continuous or discontinuous potentials. $\mathrm{J}$ Chem Phys 123:134703

63. van Krevelen DW (1992) Properties of polymers. Their correlation with chemical structure, their numerical estimation and prediction from additive group contributions, 3rd edn. Elsevier, Amsterdam, p 516

64. Oh JK, Yang J, Tomba JP, Rademacher J, Farwaha R, Winnik MA (2003) Molar mass effect on the rate of polymer diffusion in poly(vinyl acetate-co-butyl acrylate) latex films. Macromolecules 36:8836-8845

65. Wu J, Tomba JP, Winnik MA, Farwaha R, Rademacher J (2004) Effect of gel content on polymer diffusion in poly(vinyl acetate-codibutyl maleate) latex films. Macromolecules 37:4247-4253
66. Farinha JPS, Wu J, Winnik MA, Farwaha R, Rademacher J (2005) Polymer diffusion in gel-containing poly(vinyl acetate-co-dibutyl maleate) latex films. Macromolecules 38:4393-4402

67. Tomba JP, Portinha D, Schroeder WF, Winnik MA, Lau W (2009) Polymer diffusion in high-M/low-M hard-soft latex blends. Colloid Polym Sci 287:367-378

68. Hess B, Kutzner C, van der Spoel D, Lindahl E (2008) GROMACS 4: algorithms for highly efficient, load-balanced, and scalable molecular simulation. J Chem Theory Comput 4:435-447

69. Humphrey W, Dalke A, Schulten K (1996) VMD: visual molecular dynamics. J Mol Graph 14:33-38 